\title{
Modeling and analysis of solar wind generated contributions to the near-Earth magnetic field
}

\author{
S. Vennerstrom ${ }^{1}$, T. Moretto ${ }^{2}$, L. Rastätter ${ }^{2}$, and J. Raeder ${ }^{3}$ \\ ${ }^{1}$ Danish National Space Center, Juliane Maries Vej 30, DK-2100 Copenhagen, Denmark \\ ${ }^{2}$ NASA Goddard Space Flight Centre, Greenbelt, Maryland, U.S.A. \\ ${ }^{3}$ Institute for the Study of Earth, Oceans and Space, University of New Hampshire, Durham, New Hampshire, U.S.A.
}

(Received November 24, 2004; Revised August 12, 2005; Accepted August 22, 2005; Online published April 14, 2006)

\begin{abstract}
Solar wind generated magnetic disturbances are currently one of the major obstacles for improving the accuracy in the determination of the magnetic field due to sources internal to the Earth. In the present study a global MHD model of solar wind magnetosphere interaction is used to obtain a physically consistent, divergence-free model of ionospheric, field-aligned and magnetospheric currents in a realistic magnetospheric geometry. The magnetic field near the Earth due to these currents is analyzed by estimating and comparing the contributions from the various parts of the system, with the aim of identifying the most important aspects of the solar wind disturbances in an internal field modeling context. The contribution from the distant magnetospheric currents is found to consist of two, mainly opposing, contributions from respectively the dayside magnetopause currents and the cross-tail current. At high latitudes the field-aligned component is of partidular interest in connection with internal field-modelling. In the altitude regime of 400-800 km (typical for low Earth orbit satellites) the ionospheric currents are found to contribute significantly to the disturbancance, and account for more than $90 \%$ of the field-aligned disturbance. The magnetic disturbance field from field-aligned currents (FACs) is basically transverse to the main field, and they therefore contribute with less than $2 \%$ to the disturbance in total field intensity. Inhomogeneity in ionospheric conductance is identified as the main cause of main-field disturbance in the field-aligned direction. These disturbances are associated with the ionospheric Pedersen currents, and may introduce systematic errors in internal field models.
\end{abstract}

Key words: Earth's magnetic field, MHD modeling, solar wind, ionosphere, magnetosphere, quiet conditions.

\section{Introduction}

Solar wind generated magnetospheric and ionospheric currents constitute a significant source to the magnetic field near the Earth surface. When constructing accurate models of the Earth's internal magnetic field, based on data from low-altitude satellites, it is therefore a major problem to eliminate these effects. Even during quiet conditions these currents exist and may introduce significant noise as well as systematic errors in the internal field models. One way to circumvent this is to include the external variations in a simultaneous modelling of the internal and external field, the so-called comprehensive modeling (Sabaka et al., 2002, 2004). In the comprehensive approach the solar wind generated currents have so far been represented by a set of external spherical harmonic coefficients varying with local time and season. Non-periodic variations in the currents caused by UT-variations in the solar wind conditions have, however, only been included by a contribution to the 1 st order external coefficients parametrized by the geomagnetic ringcurrent index Dst (Sabaka et al., 2002, 2004), i.e. by an axial symmetric contribution.

A possible next step towards more accurate internal field

Copyright (c) The Society of Geomagnetism and Earth, Planetary and Space Sciences (SGEPSS); The Seismological Society of Japan; The Volcanological Society of Japan; The Geodetic Society of Japan; The Japanese Society for Planetary Sciences; TERRAPUB. models will therefore be to include non-axially symmetric, time varying magnetospheric contributions as well as high-latitude ionospheric disturbances in the comprehensive modeling approach. The future multi-satellite mission Swarm, planned to be launched in 2009 , provides a unique opportunity to attempt a more accurate data-based modeling of the solar wind generated disturbances.

In the present study a global MHD-model of solar wind magnetosphere interaction, coupled with an ionospheric model, is used to simulate the solar wind generated disturbances during quiet conditions at Swarm altitudes (i.e. 400$800 \mathrm{~km}$ ). The model provides a physically consistent and divergence-free set of current density at a 3D grid including both magnetospheric and ionospheric currents. With the important exception of the magnetospheric ring-current, the model simulates all the major solar wind generated currents in the magnetosphere and ionosphere. A proper description of the effect of the magnetospheric ring current is highly relevant for the improvement of internal field modeling, but the global MHD models cannot, at their present stage, provide a realistic simulation of the ring current and its time variations. Consequently the effect of the ring current is not included in the present study. Further the global MHD models only include ionospheric currents which are directly generated by solar wind magnetosphere interaction processes. External disturbances caused by current systems associated with the ionospheric dynamo (i.e. $S_{q}$ etc.), there- 
fore, are also not included. The effect of the $S_{q}$ system has also already been successfully incorporated in the comprehensive modeling (Sabaka et al., 2002, 2004).

The model is briefly described below and in more detail in an accompanying paper (Moretto et al., 2006), where the model results also are compared to observations. In the following we will analyze the simulated field, by separately estimating and comparing the magnetic field contribution from the various parts of this current system, such as the magnetopause currents, the cross-tail current, the fieldaligned currents and the high-latitude ionospheric currents. Physically the ionospheric currents are a combination of two parts, the Pedersen currents flowing in the direction of the electric field and the Hall current flowing perpendicular to the electric field. Addressing the physics of the ionospheric currents, it is therefore also interesting to estimate the separate contributions from these two currents. In addition the Swarm satellites will carry instruments to measure the electric field, and will therefore open the possibility to combine electric and magnetic field measurements in the modeling.

Solar wind generated disturbances are generally complicated and arise from a number of related current systems and processes. No physical or empirical model yet exists which can accurately predict the magnetic disturbance at a given time and place. The main objective of the present analysis is thus to identify those aspects of the solar wind generated disturbances which are most important for the derivation of the internal field using low-altitude satellite measurements. As such it may serve as a basis for a future effort to improve the external field modeling in an internal field modeling context.

\section{Model}

\subsection{The global MHD model}

The used model is the so-called Open Geospace General Circulation Model (GGCM), which was originally developed as a MHD model of Earth's magnetosphere (Raeder et al., 1995, 1998; Raeder, 2003). The model solves the ideal MHD equations in the magnetosphere, and includes a magnetosphere-ionosphere coupling module that not only maps the field-aligned currents into the ionosphere and the electric potential back into the magnetosphere, but also computes electron precipitation parameters and the ionospheric Hall and Pedersen conductivities using empirical relations in a self-consistent manner (Raeder et al., 1998; Raeder, 2003).

The magnetospheric part solves the MHD equations on a stretched Cartesian grid, spanning $288 R_{E}$ in the sunward/antisunward direction and $96 R_{E}$ in the perpendicular directions. The Earth magnetic field are simulated by a fixed dipole, and the solar wind variations are applied as boundary condition. In the simulation results used for this study the dipole tilt was set to zero. The model output are from the implementation of the code run at the Community Coordinated Modeling Centre (CCMC) at NASA Goddard Space Flight Centre. The run was performed as a so-called run on request, and the modeling results are available at the internet on the address: http://ccmc.gsfc.nasa.gov/ under run number Susanne_Vennerstrom_012804_1.
Field-aligned currents (FAC) are calculated close to the inner boundary of the magnetospheric part of the simulation (at $3.7 R_{E}$ ) and are used as input to solve the ionospheric potential equation. The field-aligned currents are mapped from the inner boundary of the magnetopheric part of the simulation into points in the ionosphere along dipole field lines. The real magnetosphere differs to some extent from this simple picture, both because the Earth's internal field is not entirely dipolar, and because the external currents produce magnetic perturbations perpendicular to the main field, which will distort the mapping.

The polar ionosphere is treated as a two-dimensional spherical shell in the altitde $90 \mathrm{~km}$, and the ionospheric current is determined from the FAC and the ionospheric conductance through determination of the electric field by the relations:

$$
\begin{gathered}
\nabla \cdot \underline{\underline{\Sigma}} \cdot \mathbf{E}=-j_{\mathrm{par}} \sin I \\
\mathbf{J}_{\text {horiz }}=\left(\begin{array}{c}
j_{\vartheta} \\
j_{\lambda}
\end{array}\right)=\underline{\underline{\Sigma}} \cdot \mathbf{E}=\underline{\underline{\Sigma}} \cdot\left(\begin{array}{c}
E_{\vartheta} \\
E_{\lambda}
\end{array}\right)
\end{gathered}
$$

$\underline{\Sigma}$ is the ionospheric conductance tensor, determined by the Hall and Pedersen conductance $\Sigma_{H}$ and $\Sigma_{P}, \mathbf{E}$ is the ionospheric electric field and $I$ is the magnetic field inclination angle (Kelley, 1989; Raeder et al., 1998). The ionospheric Hall and Pedersen conductances play a large role in determining the ionospheric electrodynamics of the model. In the implementation of the model used for this study, they are computed from empirical formulas. The conductance are proportional to the ionospheric electron density (mostly dominated by the E-region), which is mainly determined by solar EUV irradiance and precipitation of magnetospheric electrons. The effect of electron precipitation is modeled as a diffuse contribution from pitch angle scattering of hot magnetospheric electrons, and a discrete contribution from accelerated auroral electrons. The former contribution is estimated from the magnetospheric electron density and temperature, while the latter is calculated from the parallel potential drop, which in turn is parametrized mainly by the field-aligned current density. The empirical formulas used to derive the conductances can be found in Raeder (2003) (see also the accompanying paper Moretto et al., 2006). Figure 1 shows the ionospheric Pedersen conductance at two different times during the model run. The figure shows the northern polar ionosphere seen from above in a (mag. lat., MLT)-coordinate system centered on the geomagnetic pole. The day-night gradient in conductance due to the gradient in solar EUV flux are clearly seen, as well as the night-side auroral enhancement in conductance due to particle precipitation. It should be noted that observational evidence suggest that both during quiet and disturbed conditions soft electron precipitation in the polar cusp and cleft enhances ionospheric conductivity locally in this region (Hardy et al., 1985, 1987). This is observed both during quiet and disturbed conditions, but as can be seen from Fig. 1 this effect is not contained in the model. The effect, however only comprise about $20-30 \%$ of the modeled EUV conductivity in the region.

The most important model output in the present context is the current density on a three dimensional spherical grid, 

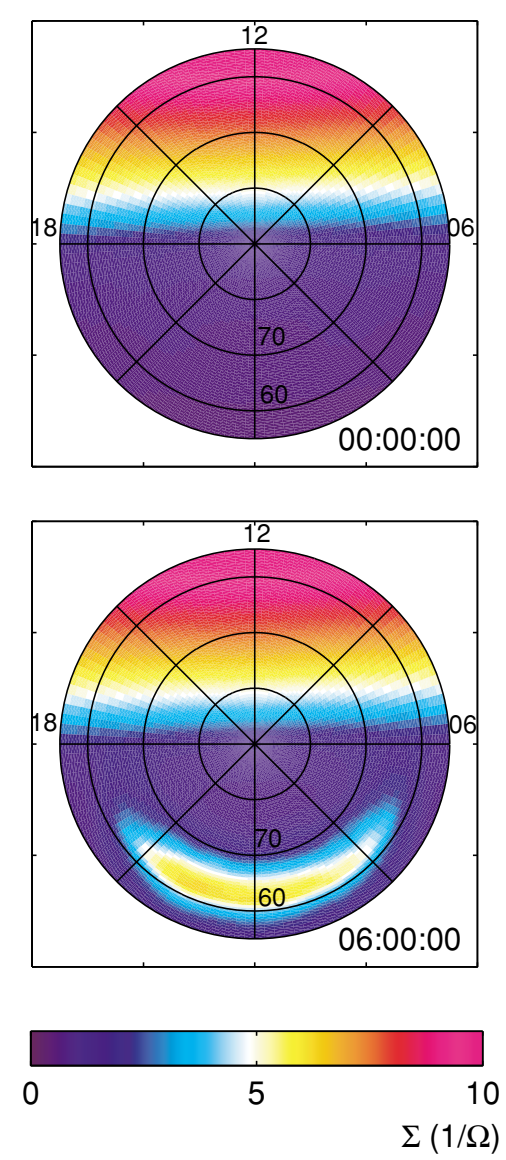

Fig. 1. Ionospheric Pedersen conductance during two time instants of the model run.Top: At the beginning of the simulation where no magnetosperic precipitation has yet developed. The shown conductivity is therefore solely due to EUV irradiance. Bottom: Later in the simulation, where the contribution from auroral precipitation is clearly visible at the night-side.

given for each time step in the simulation. The grid spans from the lowest grid points in the altitude of $90 \mathrm{~km}$ and out to $20 R_{E}$. The ionospheric currents flow in the lowest altitude bin at $90 \mathrm{~km}$. It consists of $142 \times 129 \times 128$ bins in the $(r, \theta, \phi)$ directions respectively. The bins are uniform in the $\theta$ and $\phi$ direction, but have increasing separation between grid-points in the $r$-direction. For more details in the description of the model see also the accompanying paper (Moretto et al., 2006).

2.2 The model current system during quiet conditions

The model provides physically consistent and divergence free sets of magnetopause currents, cross-tail current, fieldaligned currents flowing from the magnetosphere to the polar ionosphere and associated polar ionospheric currents. The effect of the magnetospheric ring current was not studied explicitly, because the global MHD models cannot provide a realistic simulation. Between the inner boundary of the model magnetosphere (at $3.7 R_{E}$ ) and the ionosphere it is assumed in the model that only the field-aligned currents exist. The simulated high-latitude currents and the associated magnetic perturbation have been found to correspond nicely with observations from the Ørsted and CHAMP satellites (Moretto et al., 2006). This is particularly the case during northward IMF conditions, with which



Fig. 2. Distribution of UT-hours with $K_{p} \leq 1^{+}$as a function of the hourly average value of the IMF $B_{Z}$ component for the interval.

we are primarily concerned here.

Geomagnetic activity is influenced by the combination of a wide variety of conditions in the solar wind and magnetosphere, but the single most decisive parameter is the north-south component $\left(B_{Z}\right)$ of the interplanetary magnetic field (IMF). Keeping all other parameters at average values, a small, positive (northward) IMF $B_{Z}$ component generally will produce very low levels of geomagnetic activity. A common criteria for identifying geomagnetic quiet intervals is a small value of the $K_{p}$-index. Figure 2 shows the distribution for the year 1988 of all UT-hours with $K_{p}$ values less than or equal to $1^{+}$as a function of the average value of the IMF $B_{Z}$ during the interval. It is seen that quiet intervals generally occur for a range of $B_{Z}$-values between appr. $+5 \mathrm{nT}$ and $-2 \mathrm{nT}$. Consequently, a slow variation of $B_{z}$ through these values was selected as solar wind input for the MHD-run. The IMF $B_{Z}$ component thus varied linearly from $+5 \mathrm{nT}$ to $-5 \mathrm{nT}$ through an eight hour interval, while all other solar wind input parameters was set to be constant at a value close to their average value (Solar wind speed 400 $\mathrm{km} / \mathrm{s}$, density $7 \mathrm{~cm}^{-3}$, temperature $140000 \mathrm{~K}$ and the IMF $B_{Y}$ and $B_{X}$ components zero). Here we will focus on the results from the most quiet part of the run, i.e. for the first six hours where $B_{Z}$ varied between $+5 \mathrm{nT}$ and $-2 \mathrm{nT}$.

Figure 3 shows the magnitude of the current density in the distant magnetosphere at the two extremes of the investigation when IMF $B_{Z}$ was $+5 \mathrm{nT}$ (left) and $-2 \mathrm{nT}$ (right). The current density is shown in the plane containing the noon-midnight meridian. Two intense current regions are seen, the day-side magnetopause and the tail plasma-sheet. Both are seen to change with the IMF. For positive $B_{Z}$ the dayside magnetopause currents are most intense pole-ward of the cusp region where the magnetospheric magnetic field and the IMF are anti-parallel and magnetic merging occur. For negative $B_{Z}$ the Earth magnetic field and the IMF are anti-parallel in the sub-solar region, and the most intense currents are observed here. In concert with the building up of magnetic flux in the tail associated with the dayside magnetic merging on closed field-lines, the tail-current is clearly increasing during the course of the run. The tail current is therefore significantly stronger for negative $B_{Z}$ and also closer to the Earth. 



Fig. 3. Magnitude of the model current density of the distant magnetospheric currents at two different times during the model run. At the time $t=00: 00$ when $B_{Z}=5 \mathrm{nT}$ and at the time 06:00 when $B_{Z}=-2 \mathrm{nT}$.


Fig. 4. Horizontal ionospheric sheet current densities (arrows) and vertical current densities $J_{r}$ (colorscale) in the altitude $400 \mathrm{~km}$ at three selected times during the run. Blue currents are directed inward and red currents outward.

Figure 4 illustrates the simulated horizontal and fieldaligned currents in the polar region at three times during the simulation. The three times are at the start of the run ( $t=00: 00)$ when IMF $B_{Z}$ is significantly positive, at the time $t=03: 00$ when it is slightly positive, and at time $t=06: 00$ when it is slightly negative. It is clearly seen how the character of the current system changes from intense but localized currents in the dayside near-pole region, to a more distributed current system with more intense currents at lower latitudes. The former currents are the so-called Northward $B_{Z}$ (NBZ) currents, which are driven by reconnection between the IMF and magnetospheric field lines poleward of the cusp-region. The latter distribution, meanwhile, corresponds to build-up of the region 1 currents associated with reconnection near the subsolar magnetopause.

The ionosperic current is the sum of two contributions, the Pedersen current flowing parallel to the electric field, and the Hall current flowing at right angles to the field. These two contributions are illustrated sepaprately for the time $t=00: 00$ in Fig. 5. The distinction may show up to be important since the Swarm satellites will carry instruments to measure the electric field.

It is important to note at this point that the real magneto- sphere in several respects differs from the smoothly varying and symmetric currents produced by the model. The strong dawn-dusk symmetry displayed in Figs. 4 and 5 is rarely observed, except as a statistical average (see for example Friis-Christensen et al., 1985; Papitashvili et al., 2002). The symmetry is mainly due to the choice of IMF $B_{y}=0$ in the model input (see for example Vennerstrom et al., 2005). However, although relevant, the effect of IMF $B_{y}$ is complicated and beyond the scope of the present study. Likewise, the smoothly varying largescale structure of the FACs seen in Fig. 4 is only a valid description in a statistical sense. At any given instant the real FACs at LEO-altitudes is structured in many narrow channels of intense current. Finally, the addition of dayside conductance due to soft electron precipitation in the cusp would of course also alter the currents displayed in Figs. 4 and 5 to some extent.

\subsection{Magnetic field computation at Swarm altitudes}

In the global MHD-model the magnetic field is calculated at the three dimensional grid at each timestep, but only in the region where the MHD-equations are used, i.e. down to 3.7 $R_{E}$. The magnetic field at Swarm altitudes has to be calculated from the three-dimensional current density. In the present study this is done by a direct integration of Biot- 



Fig. 5. Ionospheric Hall and Pedersen currents at the time $t=00: 00$ during the run.

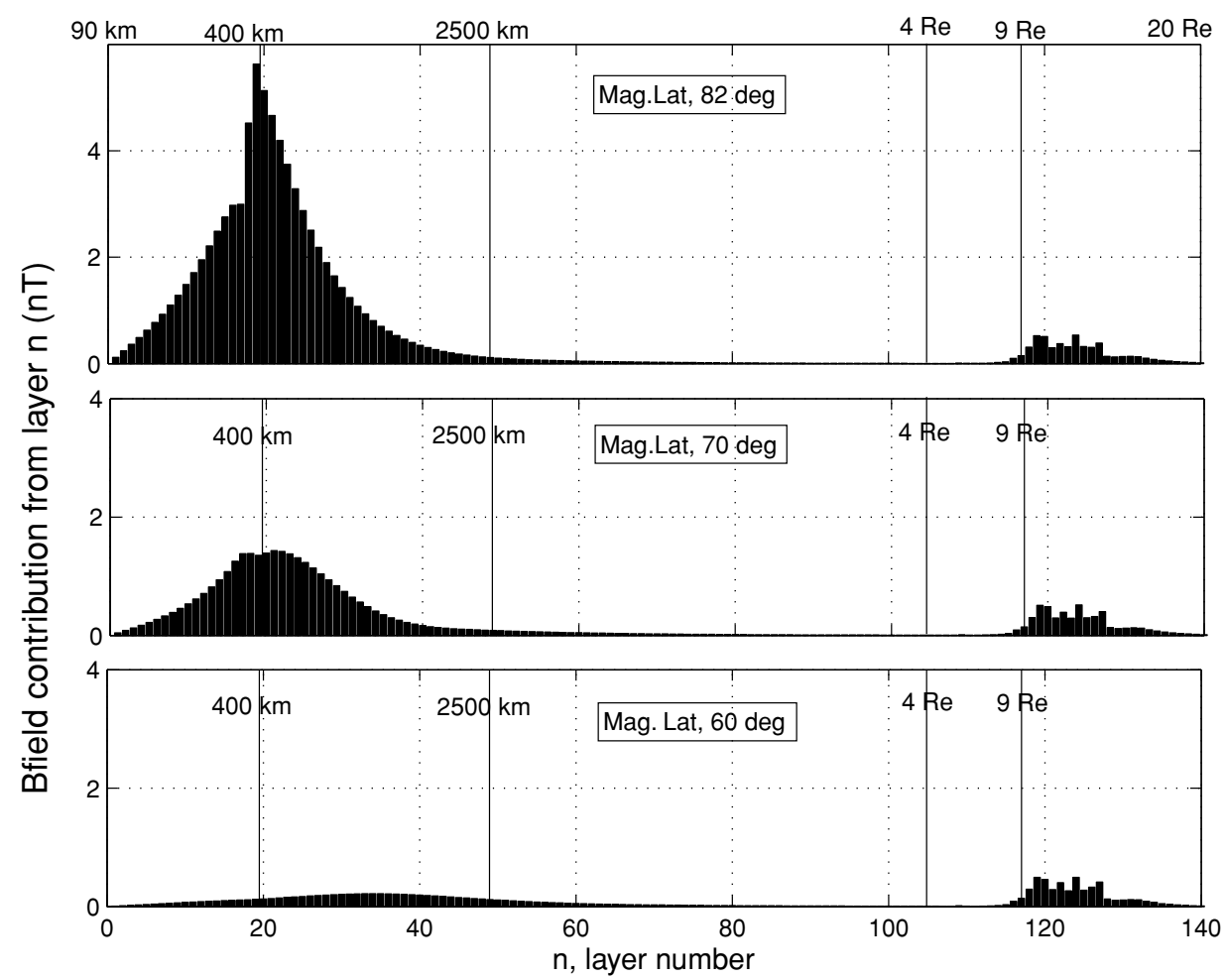

Fig. 6. Magnetic field contribution from each $r$-layer as a function of layer number, at three different points in the altitude $400 \mathrm{~km}$ at $11 \mathrm{MLT}$ and at the latitudes 82, 70 and 60 degrees. The computation was made for the time $t=00: 00$. Vertical lines show the altitude of selected layers.

Savart's law:

$$
\mathbf{B}(\mathbf{r})=\frac{\mu_{0}}{4 \pi} \int \frac{\mathbf{J}\left(\mathbf{r}^{\prime}\right) \times\left(\mathbf{r}-\mathbf{r}^{\prime}\right)}{\left|\mathbf{r}-\mathbf{r}^{\prime}\right|^{3}} \mathrm{~d} V^{\prime}
$$

over the previously described spherical grid. $\mathbf{J}$ is the current density, $\mathbf{r}$ the position vector at the point where the field is computed and $d V$ is a differential volume element. In practice the integral is evaluated numerically as the sum of the contributions from each bin in the $3 \mathrm{D}$-grid. In order to ensure an accurate estimation of the field, bins contributing more than $1 \mathrm{nT}$ to the field were subdivided into a number of sub-bins before the integration was performed. This is particularly important for bins close to the grid point at which the field is computed. When using this direct integration to compute $\mathbf{B}$ it is straightforward to assess and compare the contributions from the various parts of the current system, such as the magnetopause currents, the tailcurrents, the field-aligned currents and the ionospheric Hall and Pedersen currents.

\section{Results and Discussion}

We start by examining the relative size of the contributions from each r-layer in the spherical grid. The result will obviously depend on the location at which the field is computed. Figure 6 shows the contribution from each layer as a function of layer number, evaluated for a satellite altitude of $400 \mathrm{~km}$ at three different positions: inside the intense FAC-region, at its equatorward rim and well equatorward of the FAC's (see Fig. 4). The contribution from the bottom layer, i.e. the ionospheric currents has been omitted from the Figure, because the ionospheric currents produce a magnetic perturbation that greatly exceeds the contribution from 



Fig. 7. The magnetic field intensity due to ionospheric, field-aligned and distant magnetospheric currents, at two different altitudes and at three different latitudes at 11 MLT. The computation was made for the time $t=00: 00$. Top: $400 \mathrm{~km}$ 's altitude, Bottom: $800 \mathrm{~km}$ 's altitude.

any other single $r$-layer. The relative contributions from the ionospheric currents, the FACs and the magnetospheric currents are shown at Fig. 7. From Fig. 6 it can be seen that two maxima exist in the distribution, one associated with the FAC and one associated with the distant magnetospheric currents beyond about $9 R_{E}$. It therefore seems natural to divide the further study into separate examinations of the effect of the distant magnetospheric currents and the effect of the FAC's. Apparently the contribution from the FAC becomes insignificant beyond approximately 2500 km's altitude. At the lowest latitude the maximal contribution from the FAC's is from layers significantly above the satellite, presumably due to the bending of the field-lines.

In Fig. 7 the total contribution from the FAC's and from the distant magnetospheric currents have been computed, and the contribution from the ionosphere has been added. In addition the contribution has been evaluated at two different altitudes. Near the FAC's, i.e. at 70 and 80 degrees latitude, the FAC's are clearly the most important source, but the contribution from the ionospheric currents is definitely also significant. At $400 \mathrm{~km}$ 's altitude and at the rim of the FAC's (70 degrees latitude) the magnetic field due to the ionospheric currents are of the same size as that of the FAC's, and even deep within the FAC-region the ionospheric currents contribute with more than one third of the FAC's. Increasing the altitude by a factor of two has little effect on the FAC contribution, while the ionospheric contribution decreases by a factor of $\sim 2-3$ depending on the location relative to the most intense currents. Not unexpectedly, the contribution from the distant magnetospheric currents is the smallest, but almost independent of latitude. In the following we will examine these contributions in more detail.

\subsection{Contribution from distant magnetospheric cur- rents}

Although the contribution from the distant magnetospheric currents is relatively small it may nevertheless be important, because it is a global disturbance and not confined to high latitudes. Figure 8 shows global maps of the three components of the disturbance field, at the two times of the simulation where the IMF $B_{Z}$ is $+5 \mathrm{nT}$ and -2 nT respectively. Figure 3 shows the corresponding magnetospheric currents. Present comprehensive models of the magnetic field near the Earth includes distant magnetospheric currents, but do not include non-periodic timevariations associated with a time-varying solar wind. The results of Fig. 8 indicates that this is not a bad approximation, for the low levels of activity chosen for this study. Although substantial changes are observed in the current distribution (Fig. 3) the resulting disturbance field seems to change very little. Somewhat surprisingly the average amplitude of the disturbance actually seem to decrease as activity is building up (left: $t=00: 00$, right: $t=06: 00$ ). In a spherical harmonic expansion the fields shown at Fig. 8 can be well represented by the two coefficients $q_{1}^{0}$, giving the axially symmetric contribution, and $q_{2}^{1}$, describing the local time dependence (cf. equation (20) Langel, 1987). The shown fields corresponds to $q_{1}^{0}=3.2 \mathrm{nT}$ and $q_{2}^{1}=-1.4$ $\mathrm{nT}$ at the time $t=00: 00$ and $q_{1}^{0}=1.7 \mathrm{nT}$ and $q_{2}^{1}=-1.7$ nT at the time $t=06: 00$.

At first glance it seems strange that the dramatic change in intensity and location of the distant magnetospheric cur- 

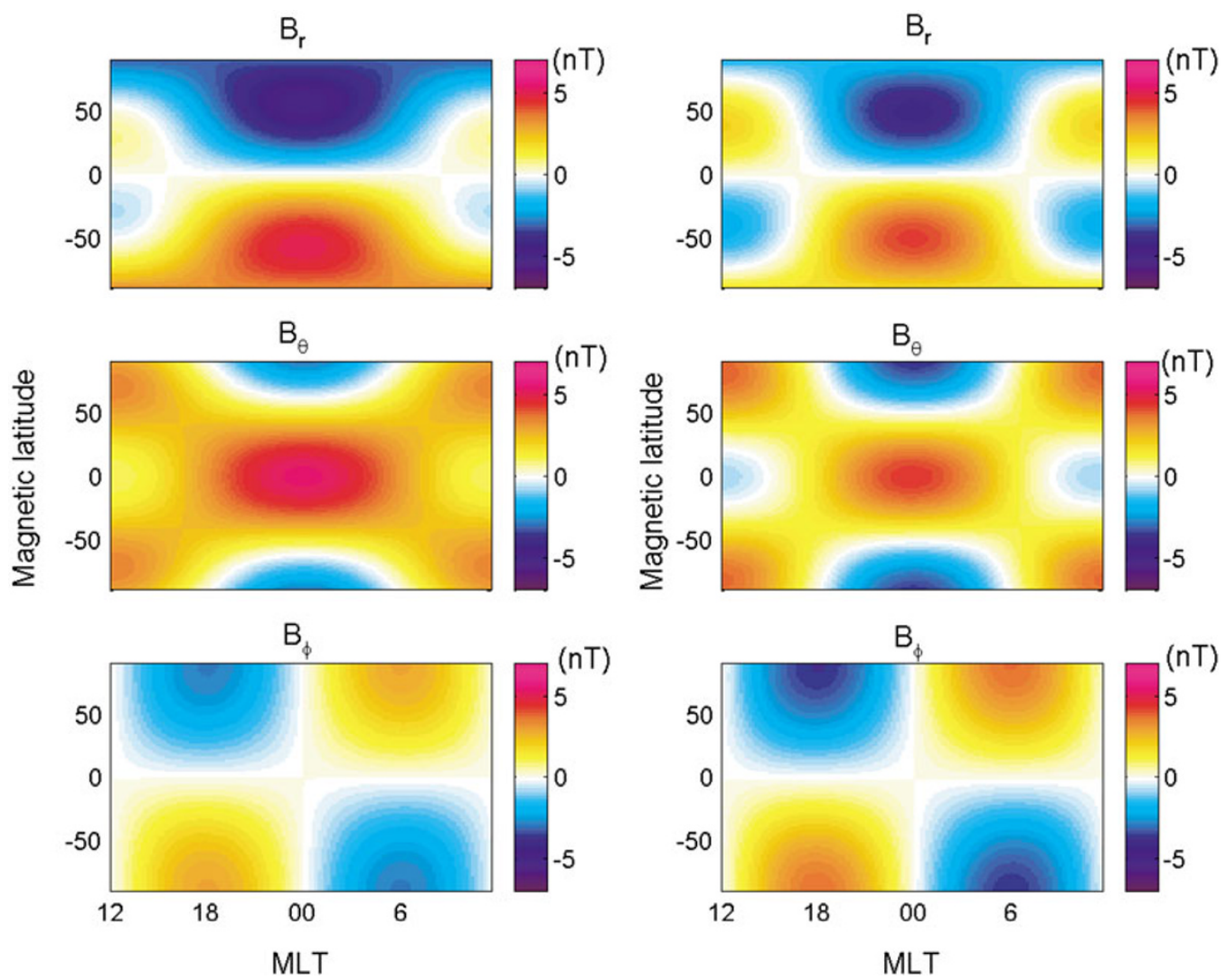

Fig. 8. Magnitude of the magnetic field disturbance due to the distant magnetospheric currents, shown as color-coded maps over the Earth in 400 km's altitude. The three components $B_{r}, B_{\theta}$ and $B_{\phi}$ are shown separately. The left panels show the disturbance field at the time $t=00: 00$ when the IMF $B_{Z}$ component was $+5 \mathrm{nT}$ and the right panels the generally more disturbed case at time $t=06: 00$ when the IMF $B_{Z}$ component was $-2 \mathrm{nT}$.
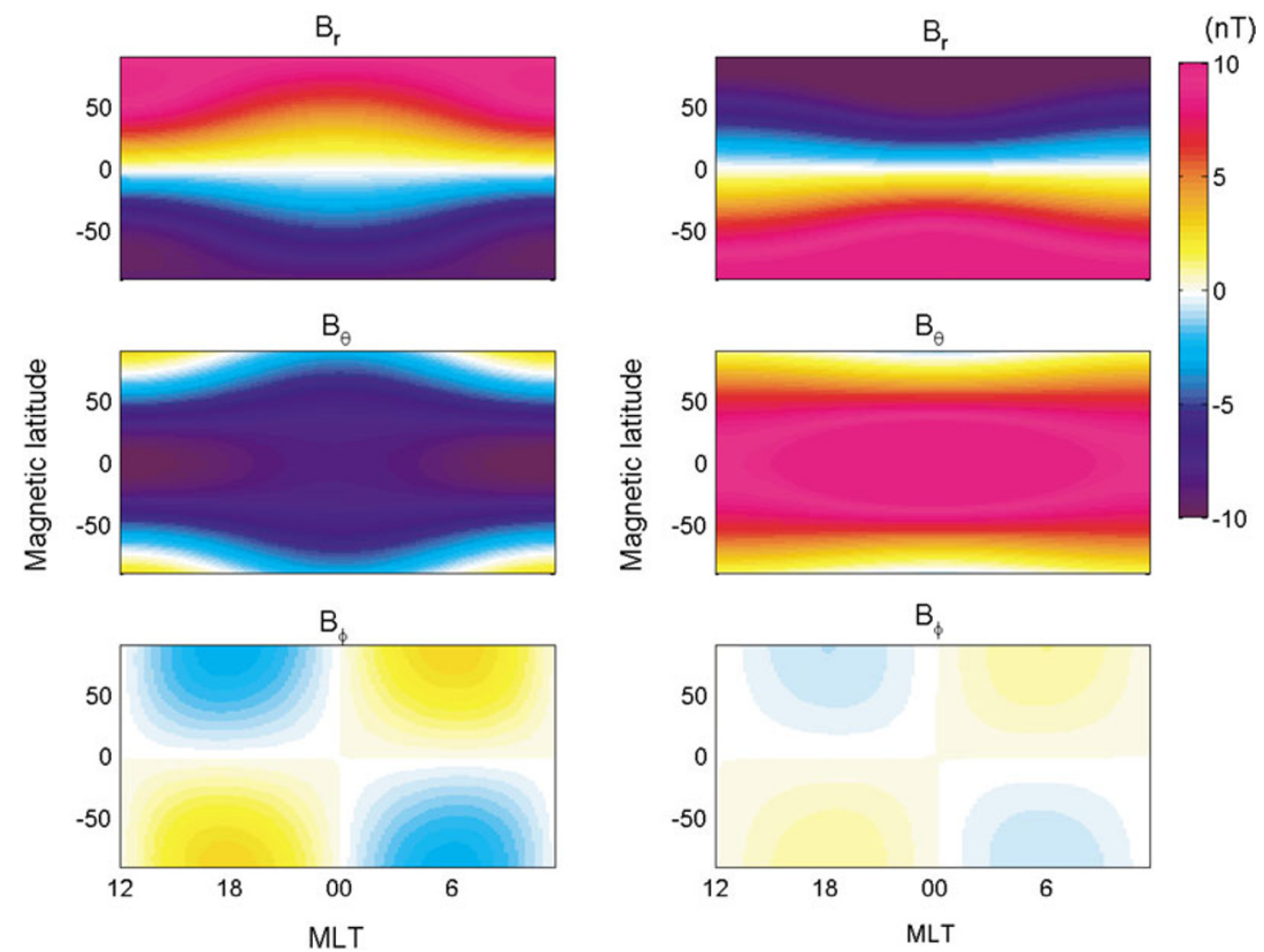

Fig. 9. The magnetic field disturbance due to the distant magnetospheric currents at time $t=06: 00$ when the IMF $B_{Z}$ component was -2 nT, shown as color-coded maps over the Earth in $400 \mathrm{~km}$ 's altitude. The three components $B_{r}, B_{\theta}$ and $B_{\phi}$ are shown separately.The left panels shows the disturbance field due to the magnetopause currents and the right panels the disturbance due to the cross-tail current. 


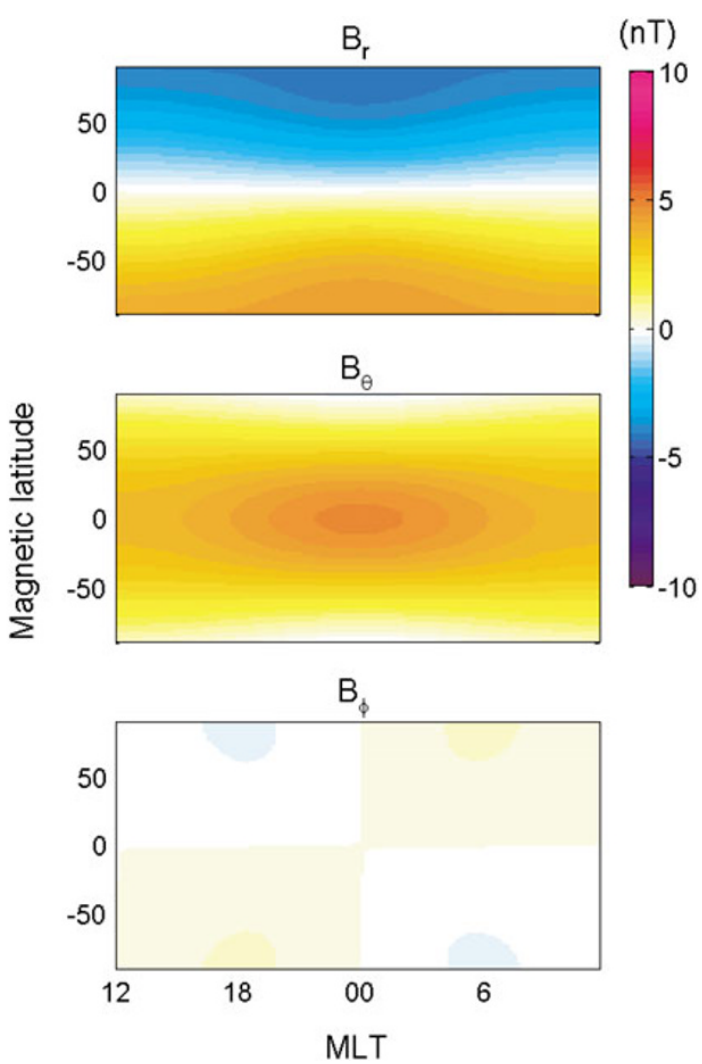

Fig. 10. Similar to Fig. 9 but for the time $t=00: 00$ when the IMF $B_{Z}$ component was $+5 \mathrm{nT}$.

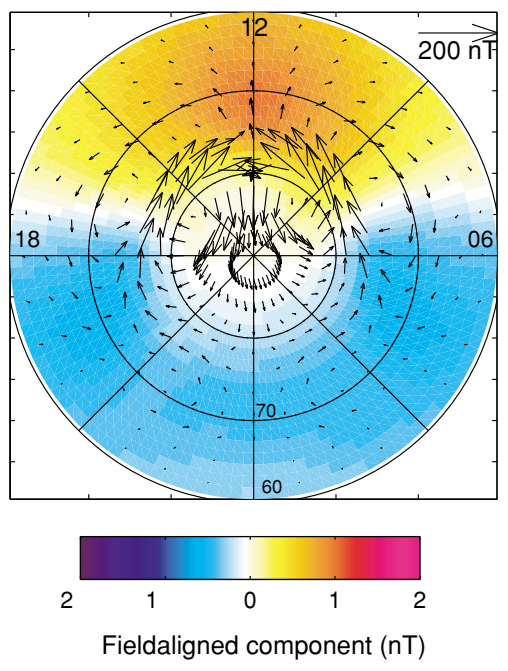

Fig. 11. Polar diagram (mag. lat., MLT) showing the magnetic field due to the field-aligned current in the northern hemisphere at the time $t=00: 00$. The arrows show the horizontal magnetic perturbation and the color-scale the field-aligned component in the altitude $400 \mathrm{~km}$. The field-aligned component is counted positive when it is parallel to the dipole-field (i.e. inward) and negative when it is antiparallel to the dipole-field (i.e. outward).

rents has so little effect at the near-Earth magnetic field. The reason, however, is that the near-Earth field is composed of two largely opposing contributions: the effect of the dayside magnetopause currents and the effect of the tailcurrent. Fig. 9 shows the separate contributions from these two current regions at the time $t=06: 00$ where the IMF $B_{Z}$ is slightly negative. The left panels show the disturbance from the magnetopause currents, and the right panels the contribution from the cross-tail current. The effect on $B_{r}$ and $B_{\theta}$ is the largest, and is evidently almost opposite for the magnetopause currents and the tail current. In the combined effect shown in the right panel of Fig. 8 large parts of the field therefore cancel.

Figure 10 shows the same division between the effect of the magnetopause currents and the effect of the tail, but for IMF $B_{Z}$ positive at the time $t=00: 00$. Here the disturbance field in the $B_{r}$ and $B_{\theta}$ components are only about half the size of the more active case. Note here also, in the left panels, the interesting disturbance near midnight in the $B_{r}$ and $B_{\theta}$ components, opposite to the disturbance near noon. This is an effect of the intense magnetopause currents at the dayside poleward of the cusp during northward IMF, which has not been described previously. Observationally it is, however also very difficult to distinguish from the effect of the tail-current.

In the real magnetosphere, where IMF changes often occur much faster, we will expect different time-delays for the day-side magnetopause current associated with dayside merging and the cross-tail current associated with the subsequent build-up of magnetic flux in the tail. In addition the dayside magnetopause currents are believed to be more sensitive to dynamic pressure changes than the cross-tail current, while the tail, in turn, can change suddenly due to substorms. We must therefore expect time variations in the magnetic field of at least the same size as the individual contributions from the magnetopause and the tail as shown in Figs. 9 and 10. Simultaneous measurements at different 


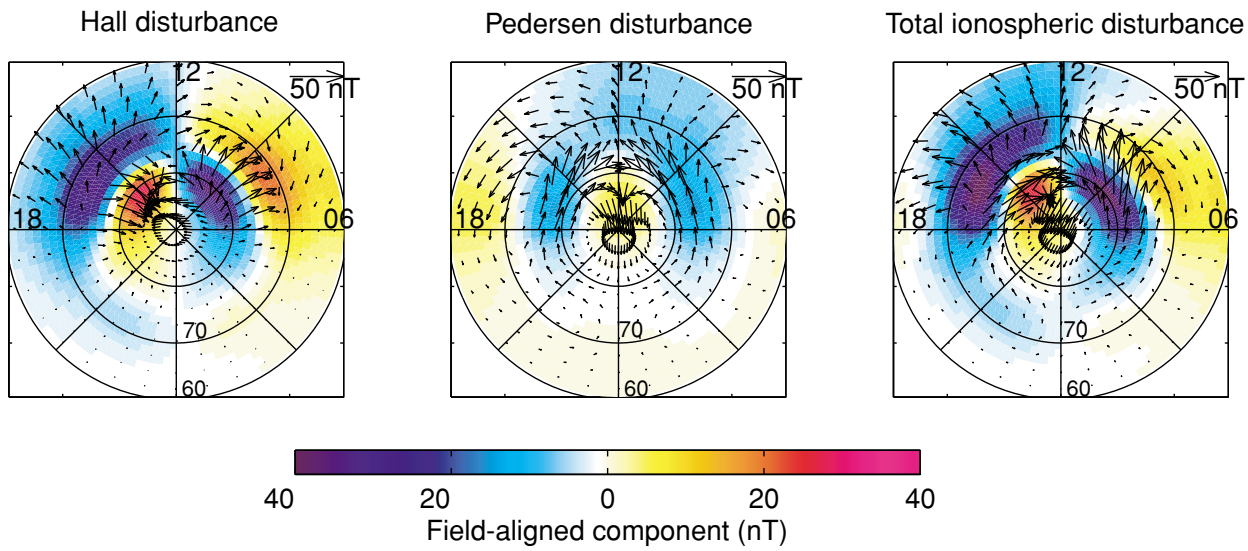

Fig. 12. Polar diagram (mag. lat., MLT) showing the magnetic field due to the Hall currents, Pedersen currents and the total ionospheric current in the northern hemisphere at the time $t=00: 00$. The arrows show the horizontal magnetic perturbation and the color-sacale the field-aligned component in the altitude $400 \mathrm{~km}$. The field-aligned component is counted positive when it is parallel to the dipole-field (i.e. inward) and negative when it is antiparallel to the dipole-field (i.e. outward).


Fig. 13. Polar diagram (mag. lat., MLT) showing the magnetic field at the surface of the Earth due to the FAC's (left) and Pedersen currents (right) in the northern hemisphere at the time $t=00: 00$. The arrows show the horizontal magnetic perturbation and the color-scale the field-aligned component. The field-aligned component is counted positive when it is parallel to the dipole-field (i.e. inward) and negative when it is antiparallel to the dipole-field (i.e. outward).

Hall disturbance

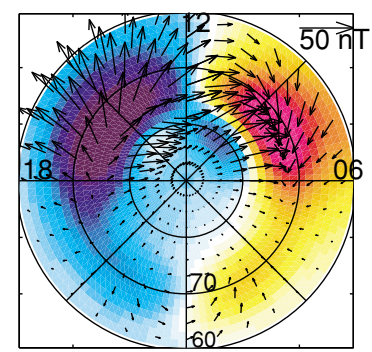

Pedersen disturbance

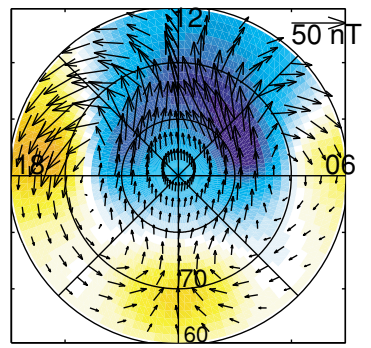

Total ionospheric disturbance

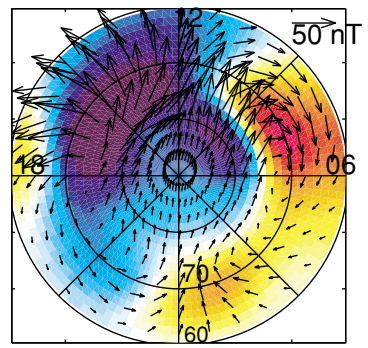

40

40

20

Fieldaligned component 20

Fig. 14. Polar diagram (mag. lat., MLT) showing the magnetic field due to the Hall currents, Pedersen currents and the total ionospheric current at the time $t=03: 00$ when $B_{Z} \sim 1 \mathrm{nT}$. The arrows show the horizontal magnetic perturbation and the color-scale the field-aligned component in the altitude $400 \mathrm{~km}$. The field-aligned component is counted positive when it is parallel to the dipole-field (i.e. inward) and negative when it is antiparallel to the dipole-field (i.e. outward).

longitudes will clearly be important when modeling these effects.

\subsection{Contribution from ionospheric and field-aligned currents}

We will now examine the high-latitude magnetic disturbance due to field-aligned and ionospheric currents in more detail. In this region the field-aligned component of the dis- turbance is of special interest in connection with internal field modeling. Due to the strong internal field, only perturbations along the internal field direction will contribute measurably to the total field intensity. Thus, a perturbation of the size of $200 \mathrm{nT}$ perpendicular to a $50000 \mathrm{nT}$ field will only alter the total field magnitude by $0.4 \mathrm{nT}$. In order to avoid the disturbing influence of the high-latitude external 
currents it is therefore customary to use only measurements of the field magnitude in the polar regions for internal field modeling.

This approach is particularly effective in eliminating the effect of the field-aligned currents. Figure 11 shows the magnetic perturbation due to the FAC's in the northern polar ionosphere at an altitude of $400 \mathrm{~km}$. The arrows show the horizontal magnetic disturbance and the color-scale the field-aligned component. Being field-aligned, the FAC's produce only a very small field-aligned component at the level of about $1 \mathrm{nT}$, which must be due to the bending of the dipole field-lines. The pattern seen in the colorscale also fits nicely into what would be expected from bending of the field-lines carrying the FAC's displayed at Fig. 4. The ionospheric currents must hence be the far most important external source of disturbances in the magnetic field magnitude in the polar region. As previously mentioned the real FACs are highly structured and less dawn-dusk symmetric. It would be interesting in subsequent studies to investigate the effect of these points on the magnetic field disturbance.

Figure 12 shows the magnetic field due to the ionospheric currents in the same format as Fig. 11. Note, though, that the color-scale has changed. The magnetic disturbance has been divided into two contributions. At the left is shown the disturbance due to the Hall currents, in the center the disturbance due to the Pedersen currents, and to the right the combined total ionospheric magnetic signature. It is clear that the Hall currents produce the largest disturbance, in particular in the field-aligned component. The field-aligned disturbance due to the Hall currents is antisymmetric with respect to the noon-midnight meridian, while the effect of the Pedersen currents is symmetric. In combination with the Hall currents the Pedersen currents therefore have the effect of introducing a dawn-dusk asymmetry in the fieldaligned component. Although the field-aligned component due to the Hall currents is larger than that due to the Pedersen currents, the contribution from the Pedersen currents may well show up to be more important in the context of internal field-modeling. If the magnetic field magnitude is sampled over a specific location with a given magnetic latitude but over all local times the contribution from the Hallcurrents will tend to cancel out due to the anti-symmetry. However, the contribution from the Pedersen currents will not, and the effect of the Pedersen currents are therefore more likely to cause systematic errors in the internal field models. It should be noted that when the IMF $B_{z}$ component changes, the latitude of the auroral oval and the accompanying current systems will also change. The tendency, for the effect of the Hall currents to cancel out, will therefore only be valid when using a large database, where each local time and geographic location is sampled for similar distributions of IMF $B_{z}$.

The algorithm used for the Biot-Savart integration can also be used to calculate the magnetic disturbance below the ionosphere at the surface of the Earth. If the field-lines are vertical and the ionospheric conductance is homogenous it was shown by Fukushima (1976) that the magnetic fields due to the Pedersen currents and the field-aligned currents cancel at the surface of the Earth. This rule, which since then has been known as Fukushimas theorem, has been widely applied in the interpretation of groundbased magnetic measurements. Figure 13 shows the magnetic field due to the model FAC (left) and Pedersen currents (right) at the surface of the Earth at the time $t=00: 00$. Evidently, in this more realistic case, with dipolar - and not straightfield-lines and non-homogenous ionospheric conductance, the two fields do not cancel. If we consider the field-aligned component, which is of special interest in the present study, the effect of the breakdown of the two assumptions can be easily separated. In the case where the field-lines are vertical and the conductance is uniform, the field-lines do not produce any vertical (field-aligned) component at the Earth (or at satellite altitude) simply because they are vertical. Consequently the vertical component from the Pedersen currents must in this case also be zero. In the present case the field-aligned disturbance due to the FAC in Fig. 13 (left) can therefore be directly translated to be the effect of the bending of the field-lines, while the field-aligned disturbance due to the Pedersen currents must be the effect of inhomogeneous conductance. Further, this must apply, not only at the ground but also at Swarm altitudes. These results therefore indicate that measurements or realistic modeling of the ionospheric conductance may be an important ingredient in future efforts to model variations of field-magnitude at high latitudes. It is also noted that the pattern of fieldaligned disturbance is very similar at the ground and Swarm altitudes, indicating that a combination of ground-based and satellite magnetic data may be straight-forward and useful.

Finally we will examine the effect of changing IMF $B_{Z}$ and of the auroral conductance enhancement. As illustrated in Fig. 1 inhomogeneity in ionospheric conductance originates from two main sources: day-night gradients in EUV-flux and auroral particle precipitation. The type of field-aligned disturbance illustrated in Fig. 12 must be expected to be typical for conductance inhomogeneites related to day-night gradient in EUV flux. Figure 14 illustrates the situation at the time $t=03: 00$ of the run, where the IMF $B_{Z} \sim 1 \mathrm{nT}$ and a small inhomogenuity in conductance is starting to develop in the nightside auroral oval. The fieldaligned Hall disturbance is also in this case basically antisymmetric with respect to the dawn-dusk meridian, while the field-aligned Pedersen disturbance is symmetric. If the Pedersen disturbance is compared to the earlier case we find that the decrease in field-intensity at latitudes between 70 and 80 degrees and the increase at lower latitudes at dawn and dusk seen in Fig. 12 is also observed here. However, due to the decline in the NBZ FAC's, associated with the decrease in the positive IMF $B_{Z}$ component, the nearpole increase in field-intensity has disappeared and has been replaced by a small decrease. Associated with the auroral conductance enhancement is now seen a nightside increase in field magnitude close to midnight.

If IMF $B_{z}$ decreases further and becomes negative activity will build up, the auroral oval and the electrojets must be expected to move towards lower latitudes and the amplitude of the disturbance will increase significantly.

The real high-latitude ionosphere seldom displays current patterns as nicely symmetric as the model currents described here. Most importantly the IMF $B_{Y}$ component introduces strong dawn-dusk asymmetries. These are, how- 
ever, believed to be antisymmetric with respect to the sign of $B_{Y}$, and could therefore be expected to cancel out when many data-points are included in the modeling, provided that the average IMF $B_{Y}=0$ for the input data. This, however, would have to be true for each local time interval. To investigate the effect of IMF $B_{Y}$, and to establish, whether the effect of the ionospheric currents on the field-aligned component found here can be found as an observational average, is an obvious candidate for future studies.

\section{Summary}

We have analyzed the magnetic disturbance at Swarm altitudes produced by a physically consistent, divergence-free model of ionospheric-, field-aligned and magnetospheric currents in a realistic magnetospheric geometry during quiet conditions. We find the following:

1) At high latitudes the main disturbance is from the FAC in the region between the ionosphere and approximately $2.5 \mathrm{Re}$. This disturbance is, however, almost perpendicular to the main field, and its contribution to variations in field magnitude almost negligible. The field-aligned component of the disturbance from the FAC's, due to the curvature of the field-lines, is estimated to be at the 1-2 nT level.

2) The ionospheric Hall and Pedersen currents both make a significant contribution to the high-latitude disturbance up to about $50 \%$, depending on latitude and the altitude of the satellite. The field-aligned contribution from the Hall current is antisymmetric around the dawn dusk meridian, while the field-aligned contribution from the Pedersen currents is symmetric. The addition of the two therefore causes a significant dawndusk asymmetry in the magnetic disturbance. The contribution from the Hall currents is larger than that from the Pedersen currents, but because of the antisymmetry the Hall contribution will tend to be zero when averaged over all local times and a large number of data-points. In terms of internal field modeling or comprehensive modeling the Pedersen currents may therefore be more important.

3) The field-aligned contribution from the Pedersen currents, which is due to inhomogeneity of the ionospheric conductance is at the $20 \mathrm{nT}$ level, during quiet conditions. This contribution can also be observed from the ground.

4) The contribution from the distant magnetospheric currents consists of two, mainly opposing parts: a contribution from the cross-tail current in the plasma-sheet and a contribution from the dayside magnetopause. The tail-contribution is dominant (around 5-10 nT) over the contribution from the magnetopause (2-7 nT). Both the location and intensity of the currents vary when IMF $B_{z}$ changes from $+5 \mathrm{nT}$ to $-2 \mathrm{nT}$, but due to the opposing nature of the currents the combined disturbance at the near-Earth changes very little in this simulation.
The results thus indicate that field-aligned magnetic disturbances at high latitudes associated primarily with ionospheric Pedersen currents and inhomogenuities in ionospheric conductance may be important in connection with internal field modeling.

The multi-satellite magnetic field mission Swarm, also carrying electric field sensors and possibly instruments to measure particle precipitation will provide a new possibility to model the effect of the ionospheric currents in a comprehensive internal/external modeling approach.

Acknowledgments. We thank the Community Coordinated Modelling Centre (CCMC) and its staff for their swift operation and assistence. Part of this study was funded by ESA contract No. 18045/04/NL/CB, The Impact of Combined Magnetic and Electric Field Analysis and Ocean Circulation effects on swarm Mission Performance.

\section{References}

Friis-Christensen, E., Y. Kamide, A. D. Richmond, and S. Matsushita, Interplanetary magnetic field control of high-latitude electric fields and currents determined from Greenland magnetometer data, J. Geophys. Res., 90, 1325-1338, 1985.

Fukushima, N., Generalized theorem for no ground magnetic effect of vertical currents connected with pedrsen currents in the uniform conductivity ionosphere, Rep. Ionos. Space Res. Jpn., 30, 35-40, 1976.

Hardy, D. A., M. S. Gussenhoven, and E. Holeman, A statistical model of auroral electron precipitation, J. Geophys. Res., 90, 4229-4248, 1985.

Hardy, D. A., M. S. Gussenhoven, and R. Raistrick, Statistical and functional representations of the pattern of auroral energy flux, number flux and conductivity, J. Geophys. Res., 92, 12,275-12,294, 1987.

Kelley, M. C., The Earth's Ionosphere, Academic Press, New York, 1989.

Langel, R. A., The main field, in Geomagnetism, edited by J. A. Jacobs, vol. 1, pp. 249-512, Academic Press, London, 1987.

Moretto, T., S. Vennerstrøm, N. Olsen, L. Rastätter, and J. Raeder, Using global magnetospheric models for simulation and interpretation of Swarm external field measurements, Earth Planets Space, 58, this issue, 439-449, 2006.

Papitashvili, V., F. Christiansen, and T. Neubert, A new model of fieldaligned current derived from high-precision satellite magnetic field data, Geophys. Res. Lett., 29, 10.1029/2001GL01,420, 2002.

Raeder, J., Global geospace modeling: Tutorial and review, in Space Plasma Simulations, edited by J. Buchner, C. T. Dunn, and M. Scholer, vol. 615 of Lecture Notes in Physics, Springer Verlag, Berlin, 2003.

Raeder, J., R. J. Walker, and M. Ashour-Abdalla, The structure of the distant geomagnetic tail during long periods of northward IMF, Geophys. Res. Lett., 22, 349, 1995.

Raeder, J., J. Berchem, and M. Ashour-Abdalla, The geospace environment modeling grand challenge: Results from a global geospace circulation model, J. Geophys. Res., 103, 14,787, 1998.

Sabaka, T. J., N. Olsen, and R. A. Langel, A comprehensive model of the quiet-time near-Earth magnetic field: Phase 3, Geophys. J. Int., 151, 32$68,2002$.

Sabaka, T. J., N. Olsen, and M. Purucker, Extending comprehensive models of the Earth's magnetic field with Ørsted and CHAMP data, Geophys. J. Int., 159, 521-547, doi: 10.1111/j.1365-246X.2004.02,421.x, 2004.

Vennerstrom, S., T. Moretto, L. Ratstätter, and J. Raeder, Field-aligned currents during northward interplanetary magnetic field: Morphology and causes, J. Geophys. Res., 110, 10.1029/2004JA010,802, 2005.

S. Vennerstrom (e-mail: sv@spacecenter.dk), T. Moretto, L. Rastätter, and J. Raeder 